\title{
Brownian flights over a fractal nest and first passage statistics on irregular surfaces
}

\author{
P. Levitz ${ }^{*}{ }^{1}$ D.S. Grebenkov, ${ }^{1}$ M. Zinsmeister, ${ }^{1,2}$ K.M. Kolwankar, ${ }^{1,3}$ and B. Sapoval ${ }^{1,4}$ \\ ${ }^{1}$ Laboratoire de Physique de la Matière Condensée, \\ UMR 7643 du CNRS, Ecole Polytechnique, 91128 Palaiseau, France \\ ${ }^{2}$ MAPMO, UMR 6628, Université d'Orléans, 45067 Orléans, France \\ ${ }^{3}$ Max Planck Institute for Mathematics in the Sciences, Inselstrasse 22, D-04103 Leipzig, Germany \\ 4 Centre de Mathématiques et de leurs Applications, \\ UMR 8536 du CNRS, Ecole Normale Supérieure, 94140 Cachan, France
}

\begin{abstract}
The diffusive motion of Brownian particles near irregular interfaces plays a crucial role in various transport phenomena in nature and industry. Most diffusion-reaction processes in confining interfacial systems involve a sequence of Brownian flights in the bulk, connecting successive hits with the interface (Brownian bridges). The statistics of times and displacements separating two interface encounters are then determinant in the overall transport. We present a theoretical and numerical analysis of this complex first passage problem. We show that the bridge statistics is directly related to the Minkowski content of the surface within the usual diffusion length. In the case of self-similar or self-affine interfaces, we show and we check numerically that the bridge statistics follow power laws with exponents depending directly on the surface fractal dimension.
\end{abstract}

PACS numbers: 05.40.Fb, 05.40.Jc, 78.55.Mb, 82.65.+r

Porous materials, concentrated colloidal suspensions, physiological organs (such as lung or kidney) are examples of systems developing large specific surfaces. They all present a rich variety of shapes and exhibit complex morphologies on a wide range of scales. In these systems, the interfacial confinement strongly influences the diffusive dynamics of Brownian particles. Although well understood for smooth interfaces, Brownian motion presents a challenging issue in the case of irregular morphologies like realistic catalytic surfaces or rough metallic electrodes while playing a crucial role in various transport phenomena in nature and industry $[1,2]$.

The interaction of a particle with a surface can be described as follows. A particle diffusing in the bulk may hit the surface. On this event, it can either react on the boundary (or be absorbed or transferred) with some probability or it can be reflected, performing a new diffusion step in the bulk. But, depending on the spatial distribution of the active sites on the surface, the consequences of the random exploration of the surface are different. If each surface element exhibits the same probability of reaction (or absorption), the overall reaction process is realized near the first hitting point on a length proportional to the inverse of the reaction probability $[2,3]$. If, on the opposite, the surface is not homogeneously reactive because the active sites for reaction are diluted on the surface, then a detailed study of Brownian bridges at long time and distance is needed. The asymptotic statistics of times and displacements between two interface encounters are then determinant to understand the overall transport. This complex first passage pro-

*Corresponding author. Tel.: +331693347 02; fax: +3316933 30 04; e-mail address: levitz@pmc.polytechnique.fr cess [4] plays a central role in thermodynamics of rough colloidal surfaces [5], or in the evaluation of the mean first exit time from a bounded domain [6]. It is also important in nuclear magnetic relaxation in complex fluids and porous media [7]. The aim of this letter is to understand how surface irregularities determine the bridges statistics.

The case of a flat interface is described by the classical Cauchy distribution [8] and was discussed in detail by Bychuk and O'Shaughnessy [9]. In this case, the probability density functions (p.d.f.) characterizing the time and distance distribution of these bridges follow Lévy statistics. The probability density $\psi(t)$ that the particle starts from a close vicinity of the interface at $t=0$ and returns to this interface, for the first time, at time between $t$ and $t+d t$ evolves as $t^{-3 / 2}$ for large $t$. At large distance, the probability density function of displacements $\theta(r)$ runs as $r^{-2}$ where $r$ is the end-to-end Euclidean distance of a Brownian bridge. The first moment (average duration or displacement of a bridge) and the second moment of the former p.d.f. are ill-defined and diverge mathematically speaking. This is a noticeable property of this specific Lévy statistics, that extends the time scale of the fluid dynamics nearby a flat interface towards the low frequency domain [7]. But, in most practical situations, irregular interfaces are encountered and the behavior associated to flat interfaces is possibly misleading.

In this letter, we first derive two general and related expressions describing the first passage statistics of Brownian bridges. Then, we propose an independent analytic validation of these relations for a large class of fractal curves embedded in 2D. Finally, we check the validity of our theoretical analysis, by an extensive numerical study in $2 \mathrm{D}$ and $3 \mathrm{D}$. 
Let us consider that a uniform and constant concentration of particles is imposed from time $t=0$ at close vicinity of the surface (Dirichlet boundary condition). Such a situation really exists in the so-called diffusion regime in electrochemistry $[10,12-15]$. Now, from time zero to time t, a diffusing particle has either come back to the source or pertains to the total number $Q(t)$ of particles which have really diffused out from the source. In such a case, $Q(t) \propto \int_{0}^{t} S\left(t-t^{\prime}\right) d t^{\prime}=\int_{0}^{t} S\left(t^{\prime}\right) d t^{\prime}$ where $S(t)$ is the survival probability of a particle starting at random nearby the interface at $t=0$. By definition, the survival probability is the probability not to hit the surface before time $t$ and $S(t)=1-\int_{0}^{t} \psi\left(t^{\prime}\right) d t^{\prime} . \psi(t)$ is then proportional to the second time derivative of $Q(t)$ whatever the geometrical complexity of the surface:

$$
\psi(t) \propto-\frac{d^{2} Q(t)}{d t^{2}} .
$$

Note that $S(t)$ being the time derivative of $Q(t)$ is simply proportional to the total particle flux at time $t$ $[5,12,13]$. The time dependent solution $Q(t)$ of the diffusion equation is, following de Gennes [10], approximately proportional to the Minkowski content (or $\epsilon$ neigbourhood) of the surface, $C_{M}(t)$, within the diffusion length $L_{D}(t) \propto t^{1 / 2}$. For a boundary of Minkowski dimension $d, C_{M}(t) \propto\left[L_{D}(t)\right]^{d_{e}-d}$ with $d_{e}$ the Euclidean dimension of the embedding space, giving $\psi(t) \propto t^{-\alpha}$ with

$$
\alpha=\frac{d-d_{e}+4}{2} .
$$

The displacement statistics $\theta(r)$ and the time statistics $\psi(t)$ are related according to:

$$
\theta(r)=\int d t \psi(t) \delta\left(\sqrt{<r^{2}(t)>}-r\right)
$$

where $\delta(\cdot)$ is a Dirac delta function and $\sqrt{\left\langle r^{2}(t)\right\rangle}$ is the mean square displacement at time $t$. Assuming that the mean square displacement $\left\langle r^{2}(t)\right\rangle$ of the Brownian motion evolves as $t$ in the bulk phase, a change of variable for the delta function in Eq. (3) gives for a fractal boundary: $\theta(r) \propto \int d t t^{-\alpha} \delta(\sqrt{t}-r) \propto 1 / r^{2 \alpha-1}$. Writing $\theta(r) \propto r^{-\beta}$, one obtains the relation between $\alpha$ and $\beta$ :

$$
\beta=2 \alpha-1
$$

Finally, from Eqs. (2) and (4), one obtains the dependence of the space exponent $\beta$ on the Minkowski dimension:

$$
\beta=d-d_{e}+3
$$

Independently from the above analysis, we can outline a direct mathematical derivation of Eq.(5). It is based on

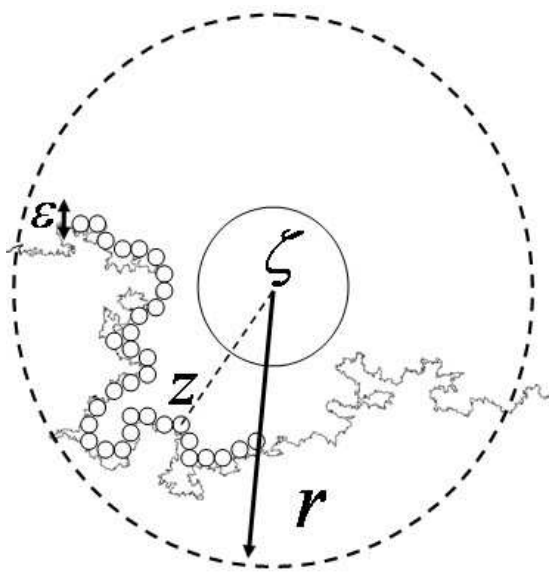

FIG. 1: Illustration of a fractal boundary within a radius $r$ with its $\epsilon$ neighbourhood. The point $z$ belongs to the $\varepsilon$ neighbourhood. This domain contains a point $\zeta$ at a distance of order $r$ from the boundary, the circle (continuous line) stands for the the ball centered at $\zeta$ of radius $r / 2$ (see text for more detail).

the argument that a particle at distance $r$ from the surface is typically captured by this surface within a scale $r$ with a finite probability (i.e. larger than some positive constant). This means that the probability to travel $r$ is, within a constant prefactor, the probability to escape to a distance $r$ from the surface. The situation is schematized in Fig. 1. This figure shows a part of a fractal boundary within a scale $r$. This domain contains a point $\zeta$ at a distance of order $r$ from the boundary. Consider the $\varepsilon$ neighbourhood of this boundary. We want to evaluate the probability $P(r, \varepsilon)$ that after having chosen with uniform law a point $z$ at distance $d(z)=\varepsilon$ from the boundary, a Brownian motion (BM) started at $z$ reaches a point $\omega$ with $d(\omega) \geq r$ before hitting this boundary. Denoting $p_{x, y}$ the probability that a BM started at $x$ reaches the ball centered at $y$ of radius $d(y) / 2$ before hitting the boundary, we may write

$$
P(r, \varepsilon) \propto\left(\frac{\varepsilon}{r}\right)^{d} \sum_{z} p_{z, \zeta} .
$$

The first factor in the right hand side describes the uniform measure on a finite set of uniformly distributed points $z$ with $d(z)=\varepsilon$. Using a well-known potential theory argument [11], one may write $p_{z, \zeta} \propto r^{d_{e}-2} G(\zeta, z)$ and $p_{\zeta, z} \propto \varepsilon^{d_{e}-2} G(z, \zeta)$, where $\mathrm{G}$ denotes the Green function of the domain. Using the equality $G(\zeta, z)=G(z, \zeta)$ and combining this with Eq.(6), we get

$$
P(r, \varepsilon) \propto\left(\frac{\varepsilon}{r}\right)^{d}\left(\frac{r}{\varepsilon}\right)^{d_{e}-2} \sum_{z} p_{\zeta, z} .
$$

But the sum is merely the total harmonic measure of the boundary, evaluated from distance $r$, with a value of or- 


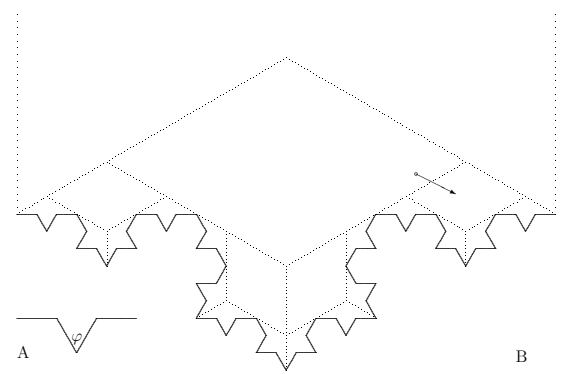

FIG. 2: Space pavement of the bulk volume which permits an efficient geometry adaptation of the fast random walk algorithm. The pavement is made of only two types on tiles: diamond and arrow head. It can be adapted to a family of Koch curves by varying angle $\varphi$ from 0 to $180^{\circ}$ in order to get a fractal dimension ranging from 1 to 2 . The distance of the random walker to the surface is computed in a sequential and hierarchical way. More details can be found in [16].

der 1. Then, $P(r, \varepsilon) \propto(\varepsilon / r)^{d-d_{e}+2}$ and $\theta(r)$, which is the first derivative of $P(r, \varepsilon)$, scales as $1 / r^{d-d_{e}+3}$. The above arguments give the outline of a complete mathematical proof which will be published elsewhere.

In order to check the validity of Eqs.(2) and (5), extensive numerical simulations using fractal boundaries have been performed. Precisely, we have investigated how $\psi(t)$ and $\theta(r)$ depend on the fractal dimension of the interface. Numerical simulations have been carried out in 2D and $3 \mathrm{D}$ embedding spaces. In $2 \mathrm{D}$, we have worked on a family of deterministic Koch curves using a geometryadapted fast random walk algorithm, recently developped [16, 17] (see Fig. 2). As a generic self-similar fractal in $\mathbb{R}^{3}$, we have studied 3D intersections of $4 \mathrm{D}$ WeierstrassMandelbrot self-affine hypersurfaces [18]. These functions are also used in 3D to generate self-affine interfaces. Starting from a random point located near the surface, an off-lattice diffusive Brownian dynamics is performed to compute the first passage statistics. In the case of selfsimilar interfaces, we found $\psi(t) \propto t^{-\alpha}$ (see Fig. 3) and $\theta(r) \propto r^{-\beta}$ for large enough $t$ and $r$. The observed dependencies of the exponents $\alpha$ and $\beta$ as functions of the fractal dimension are shown in Figs. 4 and 5. Each exponent evolves according to a single master curve gathering the numerical results obtained in $2 \mathrm{D}$ and in $3 \mathrm{D}$. One obtains approximately $\alpha=(d+2) / 2$ and $\beta=d+1$ in $2 \mathrm{D}$ and $\alpha=(d+1) / 2$ and $\beta=d$ in 3D. For self-affine interfaces, a similar trend is first observed. However a cross-over to flat interface response $(\alpha=3 / 2$ with $\beta=2)$ appears at very large $r$ and very long time $t$.

The good agreement of the numerical results with Eqs. (2) and (5) shows that the subset of random walks connecting, in the bulk, two points belonging to a confining interface is characterized by a normal dynamics associated to the scaling relation $\left\langle r^{2}(t)>\propto t\right.$. As a consequence, the Brownian dynamics in the bulk is only

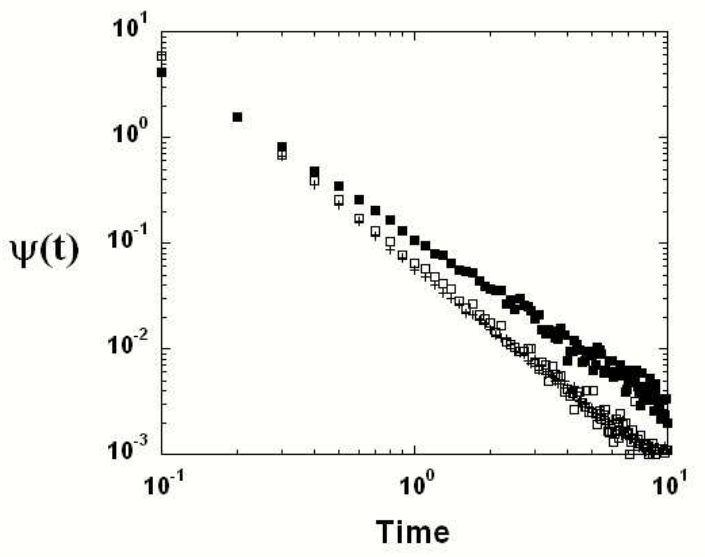

FIG. 3: Time evolution of the probability density $\psi(t)$ that the particle starting from a close vicinity of the interface at $t=$ 0 returns to this interface, for the first time, at time between $t$ and $t+d t$. Comparison between two self-similar fractal interfaces in 3D embedding space with $d=2.15$ (full squares) and $d=2.85$ (open squares), and a self-affine interface in 3D with $d=2.85$ (crosses).

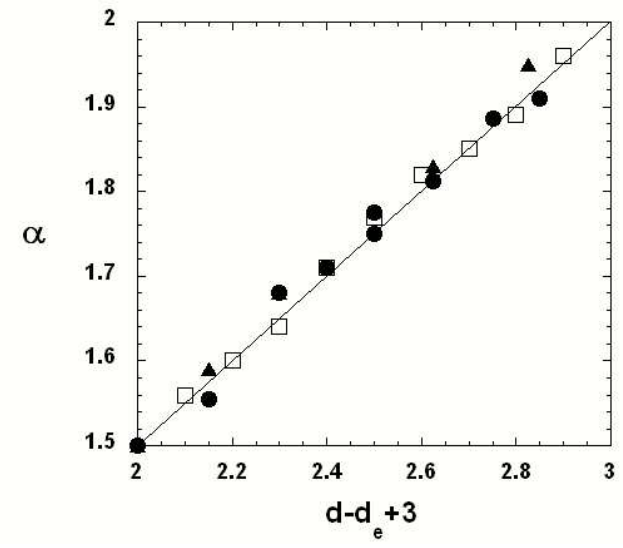

FIG. 4: Variation of the exponent $\alpha$ with the boundary fractal dimension $d$ in 2D or in 3D: self-similar curves in 2D (squares); self-similar surfaces in 3D (circles); and self-affine surfaces in 3D (triangles). The solid line follows Eq. (2).

weakly biased by the confinement induced by the surface morphology. This fact is not really surprising, as $1 \mathrm{D}, 2 \mathrm{D}$ or $3 \mathrm{D}$ random walks are characterized by the same exponent relating space and time, with only different prefactors.

Note that in this discussion the complex multifractal properties of the harmonic measure nowhere appear. The reason is that the problem we consider here is different. 


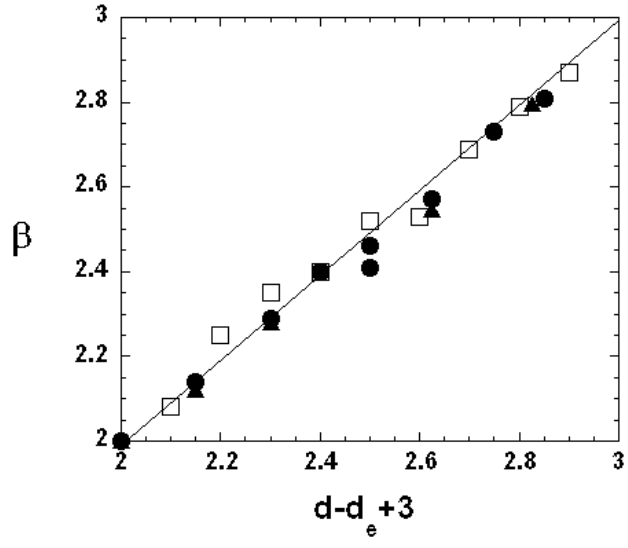

FIG. 5: Variation of the exponent $\beta$ with the boundary fractal dimension $d$ in in 2D and in 3D: self-similar curves in 2D (squares); self-similar surfaces in 3D (circles); self-affine surfaces in 3D (triangles). The solid line represents Eq. (5).

On the one hand, the departure point of the Brownian bridge is chosen uniformly on the interface. In other words, $\psi(t)$ and $\theta(r)$ are weighted by a uniform measure and not by the harmonic measure. On the other hand, we are looking for the asymptotic (large time and distance) properties of the distribution of arrival points and not for their local scaling around geometrical singularities. Consequently, although the departure and arrival points are linked by a Brownian trajectory, the multifractal exponents do not appear. A similar result was predicted by Cates and Witten [13] in the case of stationary diffusion where the exponent associated to the first moment of the incident flux involves the fractal dimension only.

Although verified here for specific fractal surfaces, Eqs. (1) and (3) are general. For instance, they could be applied to the case of diffusion nearby a mass fractal system with $1<d<2$ embedded in ordinary space (like clustercluster aggregates of smooth particles in solution). A classical behavior is expected at small distance and short time $(\alpha=3 / 2$ and $\beta=2)$, followed at large distance and time by a cross-over to $\alpha=(d+1) / 2$ and $\beta=d$. The case of a thin and very long cylinder such as DNA molecules or other similar colloidal particles is another interesting example where the embedded fluid dynamics should be sensitive to a geometrical cross-over from local to global exploration of the surface. Experiments using NMR relaxation on suspensions of long colloidal thin rods (either mineral or biological) are currently underway in our group in order to check the possibility to probe a colloidal shape, looking at the slow and confined fluid dynamics near a colloidal surface [7, 19].

In summary, we have presented two general relations describing Brownian bridges statistics near an irregular interface. These statistics have been shown to be directly dependent on the Minkowski content of the surface within the diffusion length. For a large class of selfsimilar and self-affine interfaces, we give a generalization of the Cauchy distribution to power laws with exponents showing simple dependence on the surface fractal dimension. In these situations, the Brownian dynamics in the bulk is not essentially biased by the confinement induced by the boundaries. The exponent $\beta$, corresponding to the Euclidean displacement distribution, is found to be strictly larger than 2. Consequently the mean distance for the first passage encounter is now finite in opposition with the case of a flat surface. The fact that Brownian bridges are sensitive to surface geometrical cross-overs at long time, should provide a way to probe colloidal shape [19].

The authors are grateful to Professor N. Makarov for valuable discussions.

[1] M. F. Shlesinger, J. Klafter and G. Zumofen, Am. J. Phys. 67, 1253 (1999).

[2] B. Sapoval, Phys. Rev. Lett. 73, 3314 (1994).

[3] D. S. Grebenkov, M. Filoche and B. Sapoval, Eur. Phys. J B 36, 221 (2003)

[4] S. Redner, A Guide to First Passage Processes, Cambridge University press (2001).

[5] B. Duplantier, Phys. Rev. Lett. 66, 1555 (1991)

[6] O. Benichou, M. Coppey, M. Moreau, P. H. Suet and R. Voituriez, Europhys. Lett. 70, 42 (2005).

[7] P. Levitz and J.-P. Korb, Europhys. Lett. 70, 672 (2005).

[8] W. Feller, An Introduction to Probability Theory and its Applications, John Wiley and Sons, New York (1968).

[9] O. V. Bychuk and B. O'Shaughnessy, J. Phys. II (France) 4, 1135 (1994).

[10] P.-G. de Gennes, C.R.A.S. Paris II 295, 1061 (1982).

[11] D. Jerison and C. Kenig, Adv. in Math. 46, 80 (1982).

[12] A. Maritan, A. L. Stella and F. Toigo, Phys. Rev. B. 40, 9269 (1989).

[13] M. E. Cates and T. A. Witten, Phys. Rev. A. 35, 1809 (1987).

[14] B. Sapoval, J.-N. Chazalviel, and J. Peyrière, Phys. Rev. A 38, 5867 (1988).

[15] L. Nyikos and T. Pajkossy, Electrochimica Acta 31, 1347 (1986).

[16] D. S. Grebenkov, A. A. Lebedev, M. Filoche and B. Sapoval, Phys. Rev. E 71, 056121 (2005).

[17] D. S. Grebenkov, Phys. Rev. Lett. 95, 200602 (2005).

[18] P. Levitz, Europhys. Lett. 39, 593 (1997).

[19] P. Levitz, J. Phys.:Condens. Matter 17, S4059 (2005). 